\title{
Zum Standort der Geographie als Universitätsdisziplin*
}

\section{Die Geographie als Hochschulinstitution}

\subsection{Der Wandel von Gesellschaft, Universität und Forschungstechnologie}

Die Geographie ist als Bildungsdisziplin im 19. Jahrhundert entstanden, ihre Sachinhalte gehörten zum Allgemeinwissen des Bildungsbürgertums eines liberalen Zeitalters, dem die Geographie auch vorschreiben konnte, was «Bildung» ist. Von Angehörigen der Oberschichten, dem Adel und dem Bürgertum, wurden die Geographischen Vereine gegründet, in einer Zeit, als die Kenntnis der Erde ebenso wie ihre Erforschung über Expeditionen ein Privileg der oberen Schichten darstellten. Es ist hier nicht der Platz, um die Wachablöse der Gesellschaftskonzepte und die Rückwirkung auf die Bildungsdisziplin zu diskutieren, welche in diesem Jahrhundert eingetreten ist. Sicher ist eines: Wir leben in einer Konsumgesellschaft, in - um einen Ausdruck von H.SCHELSKY zu verwenden - einem Zuteilungsstaat. Breite Bevölkerungskreise erhalten über Massenmedien in diffuser Weise ein geographisches Weltbild, dessen einzelne Komponenten schwierig zu erfassen sind, bei denen man jedoch in einem sicher sein kann, nämlich daß die Normen und Werthaltungen der Konsumgesellschaft und in zunehmendem Maße auch der Freizeitgesellschaft die Inhalte bestimmen. Das Interesse an einer "géographie de plaisir" liegt nahe. Durch die Informationen über die Massenmedien, in erster Linie das Fernsehen, werden die räumlichen Distanzen vernichtet. Ein punktuelles Wissen oft skurriler Art bleibt übrig. Hierin wurzelt übrigens das Take-off der Fallstudie in der Schuldidaktik. Die Fachwissenschaft besitzt keinerlei Kontrollfunktionen mehr über dieses Wissen, so wie sie es einst beim Bildungsbürgertum hatte. Dies führt so weit, daß die neu an die Universitäten einrückende Generation von Studenten auch nicht mehr bereit ist, sich mit dem zu belasten, was sie als Ballast empfindet, nämlich das topographische Wissen über die Erde als Ganzes, über Kontinente und Staaten.

*Vortrag, gehalten anlässlich der 100-Jahr-Feier der Schweiz. Geographischen Gesellschaft in Bern, Dezember 84.
Mit dem Wandel der Gesellschaft ist auch ein grundsätzlicher Wandel der Institution der Universitäten erfolgt (vgl. Abb. 1). Die Bildungspolitik der sozialen Wohlfahrtsstaaten hat aus den elitären Universitäten Massenuniversitäten gemacht. Universitätsorganisationsgesetze, wie in Österreich und in den meisten bundesdeutschen Ländern, haben die klar definierten

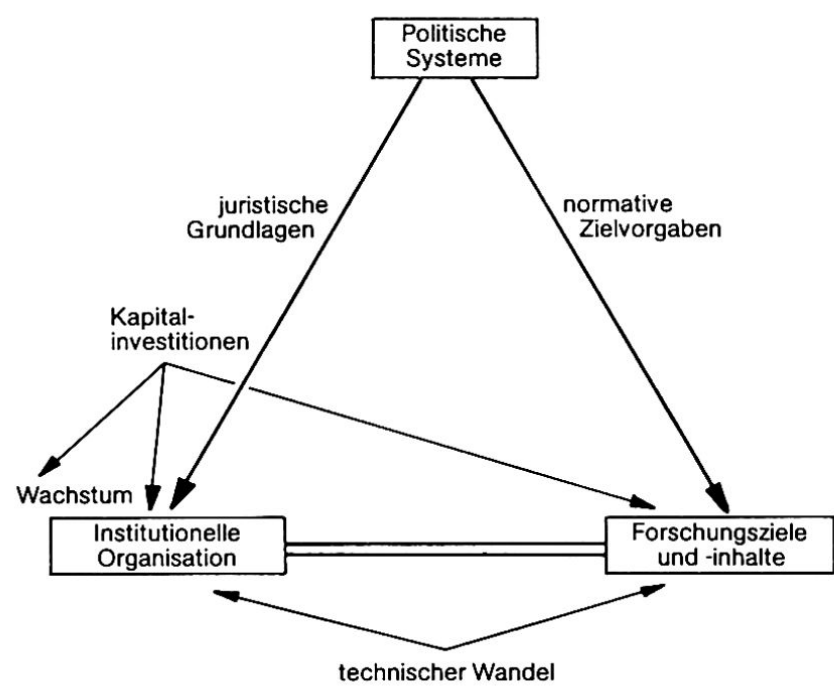

Entwurf: E. Lichtenberge

Abb. 1 Das Beziehungsdreieck von politischen Systemen, institutioneller Organisation und Forschungszielen

hierarchischen Entscheidungsstrukturen durch egalitäre, drittelparitätische Modelle ersetzt. In Österreich wurde das Berliner Modell imitiert, durch das sich unser Staat grundsätzlich von der Schweiz unterscheidet. Hierbei blieb in einer merkwürdigen Inkonsequenz aus der Zeit der HUMBOLDTSCHEN Universität die Idee des Einzelwissenschaftlers erhalten. Individualität ist nach wie vor Trumpf, auf ihr beruhen die Karrierenormen. Teamarbeit als legitime und dem technologischen Standard der analytischen Forschung

Elisabeth Lichtenberger, o. Univ.-Prof., Dr., Institut für Geographie der Universität Wien, Studienzweig Raumforschung und Raumordnung, Universitätsstr. 7, A-1010 Wien 
adäquate Organisationsform - in der Privatwirtschaft gang und gäbe - ist derzeit in den Karrierenormen der ehemaligen Artistenfakultäten nach wie vor diskriminiert. Die Umbrüche der Universitätsorganisation haben auch nichts daran geändert, daß die universitäre Laufbahn im großen und ganzen ein nach außen hin abgeschlossener und gleichsam systeminterner Reproduktionsprozeß geblieben ist. Ein Stellenwechsel zwischen Universität, öffentlichem und privatem Sektor ist - ebenfalls an den Artistenfakultäten praktisch bedeutungslos. Unabhängig von der Universitätsgesetzgebung ist in allen westeuropäischen Staaten eine Vermehrung und strukturelle Veränderung dessen eingetreten, was heute unter dem Begriff des "akademischen Personals» in Jahresberichten veröffentlicht wird. Ganz allgemein ist ferner eine Erweiterung des Aufgabenbereichs der Institute eingetreten.

Zwar ist laut Staatsgrundgesetz «Freiheit der Forschung» und "Einheit von Forschung und Lehre» in der Eingangshalle von Universitäten - wie in der Wiener Universität - noch auf einer großen Tafel angeschlagen, der man, wenn man die Wirklichkeit kennt, nur mehr die Funktion einer potemkinschen Fassade zuschreiben kann. Mit der vielgerühmten Selbstverwaltung der Universitäten haben die Institute zunehmend Verwaltungsaufgaben übernehmen müssen. Das Heraustreten aus dem vielkritisierten «elfenbeinernen Turm" hat eine Flut von Expertisen zur Folge gehabt, welche vielfach auf Kosten der Grundlagenforschung erfolgten, ohne jedoch de facto das Ansehen der Wissenschaft in der Öffentlichkeit zu vergrößern.

Es stellt sich die Frage, wie diese Vielzahl von Aufgaben verknüpft werden kann. Grundsätzlich sind zwei organisatorische Formen möglich:

1. Unter Beibehaltung der funktionellen Vielseitigkeit wechselt der einzelne im Laufe der Zeit (und des Lebens) die Aufgaben. Dies bedeutet einen Standortund Aufgabenwechsel im internen bzw. externen Informations- und Aktionssystem in täglichen, wöchentlichen, jährlichen und mehrjährigen Rhythmen. Es wäre einmal eine interessante Aufgabe, die Terminkalender von Professoren in diesem Zusammenhang einer Zeitbudget-Analyse zu unterziehen.

2. Es sind neue Idealtypen entstanden, wie die Kongreßexperten, die, in z.T. geschlossenen Circles, quer über die Erde hinweg von einer Tagungsstätte zur anderen reisen, die Vortragsreisenden, die gelegentlich eine Art Wissenschaftsjournalismus betreiben, die universitären Gesellschaftspolitiker, welche sich häufig um die Aufstockung der Budgets ihrer Institute bemühen und dadurch hineingezogen werden in die Rolle von universitären Verwaltungsbeamten. Nur eine kleine Gruppe zählt zu den Forschungsmanagern, und immer geringer wird auch die Zahl der akademischen Lehrer sensu stricto. Es sei offen ausgesprochen, $\mathrm{da} ß$ es überhaupt nur mehr wenigen gelingt, sich in institutionellen Nischen den notwendigen geistigen Freiraum für die Lebensform der Gelehrten zu erhalten, aus deren wissenschaftlicher Gemeinschaft die Universität entstanden ist.

Von entscheidender Bedeutung ist der Wandel der Forschungstechnologie, dessen Konsequenzen hier nur kurz skizziert werden können. Mit dem Eintritt in das Computerzeitalter geht auch in der Geographie das Zeitalter der Handarbeit und des Manufakturwesens einem Ende zu. Eine Technisierung der wissenschaftlichen Produktion ist möglich geworden und in Gang gekommen. Neue Institutionsformen und Strategien für wissenschaftliche Forschung zeichnen sich damit ab. Zum Verständnis der Konsequenzen dieser Entwicklung sei ein Vergleich der Industrialisierung der Sachgüterproduktion mit der Industrialisierung der wissenschaftlichen Produktion gestattet. Folgende Parallelen drängen sich auf:

Ebenso wie in den Anfängen der Konsumgüterindustrie dieselben Fertigungsziele wie im Manufakturwesen auf dem Programm standen, ebenso versucht man nun in der geographischen Forschung mittels des Einsatzes von EDV und multivariaten Techniken, die gleichen Probleme zu lösen, welche man bisher mit qualitativen Kriterien angegangen ist, wie z.B. das traditionelle Anliegen der Regionalisierung.

Wir dürfen ferner daran erinnern, daß in den Anfängen der Industrie die Produkte schlechter waren als die des traditionsreichen Gewerbes, und sie wurden auch - die Österreichisch-Ungarische Monarchie bietet hierfür ein gutes Beispiel - in weniger entwikkelte Staaten exportiert. Wir können uns daher gar nicht wundern, daß EDV-Atlanten nicht die hohen Qualitätsnormen der traditionellen Kartographie erreichen.

In der zweiten Phase der Industrialisierung ist bekannterweise das Take-off der Produktionsgüterindustrie in der klassischen Phase von Kohle und Stahl erfolgt. Neue Produkte konnten erzeugt werden. Diese Situation ist bereits vor längerer Zeit von den Naturwissenschaften erreicht worden, und in dieser Hinsicht besteht auch ein Vorsprung der Physischen Geographie gegenüber der Humangeographie. Doch hat auch letztere bei großen EDV-gestützten Forschungsprojekten Erkenntnisse erbracht, die mit den traditionellen Methoden nicht möglich gewesen wären (u. a. Gastarbeiterprojekt in Wien 1974, 1981). Zweifache Rückwirkungen der Technisierung auf die Forschung bestehen:

1. Durch den Kapitalbedarf für die notwendige Ausstattung mit teurer Hard- und Software erfolgt eine Konzentration auf wenige Institute.

2. Über internationale Programme vergrößert sich der Vorsprung der naturwissenschaftlichen Disziplinen, auch in der Geographie. Um daran partizipieren zu können, sieht sich der Einzelforscher gezwungen, einheitliche Klassifikationssysteme, Methoden und 
Fragestellungen zu verwenden. Wenn man den Grad der Internationalisierung von wissenschaftlichen $\mathrm{Er}$ gebnissen als Kriterium heranzieht, so gelangt man zur Aussage, da $\beta$ sich die Schere zwischen den naturwissenschaftlichen und den sozialwissenschaftlichen Subdisziplinen des Faches immer weiter öffnet.

\subsection{Der Einfluß der politischen Systeme: Das Beispiel der Bundesrepublik Deutschland und der DDR}

Die Veränderungen der politischen Großsysteme der Erde finden in den Forschungsstrukturen der Geographie einen geradezu modellhaften Niederschlag. In der Zwischenkriegszeit war die europäische Geographie durch zwei Modelle bestimmt, und zwar einerseits durch das französische Modell mit der absoluten Dominanz einer hochstilisierten, methodisch weitgehend perfektionierten géographie régionale, welche vor allem Großbritannien, die Iberische Halbinsel, Italien beherrscht hat, und andererseits das deutsche Modell mit der Zentrierung um das Landschaftskonzept, welches entscheidend war für den ganzen Osten und Südosten Europas. In der realen Forschungspraxis war es gekennzeichnet durch eine äußerst interessante Verkettung von Morphologie und Siedlungsgeographie als "Kerndisziplinen» mit wechselseitiger Beeinflussung von positivistischem Denken und historischer Perspektive. Ersteres wurde durch die Karrierenormen und ihre "Vorschriften" des Wechsels von der Morphologie zur Siedlungsgeographie in letztere transferiert. Abgehoben und in geringem Konnex damit vollzog sich in Nordamerika eine teilweise unabhängige, teilweise über die Schule von c. O. SAUER in Berkeley von der deutschen Landschaftsgeographie beeinflußte Entwicklung.

Die Teilung der Welt in zwei Hemisphären und die Zweiteilung Europas nach dem Zweiten Weltkrieg haben grundsätzliche Veränderungen gebracht. Die gesamte westliche Welt geriet unter den Einfluß der angelsächsischen Geographie. Die britische Geographie hat sich, begünstigt durch die Sprachgemeinschaft, sehr rasch dem Take-off der amerikanischen Analytik angeschlossen, während andererseits in Frankreich und in der Bundesrepublik Deutschland Persistenzen der oben skizzierten Modelle bis zur Gegenwart herauf bestehen.

Die Zweiteilung Deutschlands und die Zugehörigkeit zu den beiden politischen Hemisphären gestatten es, die wesentlichen forschungsmäßigen und institutionellen Unterschiede der Geographie zwischen der Bundesrepublik Deutschland und der DDR stellvertretend für andere Nationen herauszustellen.

1. Entsprechend der Zugehörigkeit zu zwei verschiedenen Kommunikations- und Informationssystemen zählt es zu den Selbstverständlichkeiten, daß einerseits in der Bundesrepublik Deutschland die angelsächsische Literatur und andererseits in der DDR die russische rezipiert wird, so daß in beiden Staaten eine gewisse nicht hinterfragte "Indoktrinierung» der Forschung, vor allem in der Humangeographie, erfolgt, und zwar einerseits über "kapitalistische Marktmechanismen» und Partizipation an wissenschaftlichen Modeströmungen, wie denen des Behaviourismus und der Zeitgeographie, und andererseits auf dem "politischen Verordnungswege» gleichsam von oben her.

Der Einfluß der politischen Doktrin auf die Wissenschaftssprache hat nicht nur in der DDR das gesamte sprachliche Design wesentlich verändert, sondern ebenso ist es im Westen des Eisernen Vorhangs aufgrund der Infiltration von zahlreichen englischen Ausdrücken, des Eindringens behaviouristischer Vorstellungen, der Übernahme einer Selbstidentifikationstheorie und -ideologie und, interessanterweise unterstützt durch die Massenmedien, des Einsickerns eines neomarxistischen Vokabulars zu einer beachtlichen Veränderung des Fachjargons gekommen. Es ist derart eine sprachliche Auseinanderentwicklung erfolgt, so daß sich selbst bei Grundbegriffen der jeweilige Sinngehalt deutlich unterscheidet. Hierzu ein Beispiel: Die Zentralörtliche Theorie von w.CHRISTALLER, implizit als konsumentenorientiertes Versorgungsmodell gedacht, enthält in der DDR-Literatur ebenso implizit die Ideologie einer produktionsorientierten Gesellschaft.

2. Entsprechend dem Vorbild der UdSSR ist die Forschung in der DDR an der Akademie der Wissenschaften konzentriert und nach einem Forschungsplan, der direkt auf den Parteibeschlüssen der SED beruht, eingestellt. Es bestehen Verträge mit den staatlichen Planungskommissionen und Planungsbehörden der Bezirke und von Berlin sowie dem Ministerium für Wirtschaft und Technik.

Selbst in einem Staat mit einer grundsätzlich liberalen Forschungspolitik wie der Bundesrepublik Deutschland besteht jedoch eine indirekte Steuerung der Forschung über die Deutsche Forschungsgemeinschaft. Diese Institution folgt letztlich der «Staatsideologie der Exportförderung». Es ist daher begeiflich, daß dank der Mittel der Deutschen Forschungsgemeinschaft die Auslandsforschung in der Bundesrepublik Deutschland seit langem die Inlandsforschung überflügelt hat. Im Gegensatz dazu steht in der DDR ebenso wie in den anderen Oststaaten die Inlandsforschung mit Abstand an erster Stelle.

3. Die Forschungsperspektiven sind in der DDR auf zwei Schwerpunkte konzentriert, nämlich ökologische Landschaftsforschung und Territorialforschung. Bei der ersteren geht es um die Kennzeichnung und Bewertung der natürlichen Ressourcen und ihre ökonomische Kategorisierung, bei der letzteren darum, "Grundlagen und prognostische Instrumente für die planmäßige räumliche Organisation gesellschaftlicher Prozesse zu liefern". 
In der Bundesrepublik Deutschland haben sich aufgrund des Wachstumssyndroms, an dem die Geographie als Universitätsdisziplin partizipieren konnte, und aufgrund der liberalen Forschungspolitik die Perspektiven der Forschung zunehmend aufgefächert. Die derzeitige Situation ist durch folgende Trends gekennzeichnet:

- eine zunehmende Verselbständigung der Teildisziplinen,

- eine immer weitere Aufspaltung derselben, d. h. das Entstehen einer immer größeren Zahl von "Geographien", die sich mit immer spezialisierteren Sachverhalten beschäftigen,

- eine wachsende methodische Spezialisierung, d.h. eine zunehmende Bedeutung des Methodenmonismus,

- eine Verlagerung der Forschung in Grenzbereiche zu Nachbardisziplinen hin, zu denen theoretische, modellhafte oder methodische Verbundschienen bestehen.

\subsection{Die Kleinstaaten Schweiz und Österreich}

Lassen Sie mich nunmehr die räumliche Sicht auf die beiden Kleinstaaten Schweiz und Österreich verkürzen. Sie weisen institutionell und forschungsmäßig gemeinsame Züge auf.

1. Die in beiden Staaten vorhandene schmale institutionelle Basis des Faches, d.h. die kleine Zahl von Universitätsinstituten und wissenschaftlichen Mitarbeitern, führte mit Notwendigkeit zu einer gewissen Monopolisierung einzelner Forschungsrichtungen durch bestimmte Persönlichkeiten.

2. Wesentlich stärker als in der Bundesrepublik Deutschland ist die Territorialität, und diese bewirkt, da $\beta$ das Forschungsspektrum des einzelnen vielfach abhängig ist vom physischen und kulturellen Umfeld der betreffenden Universitätsstadt.

3. In beiden Staaten hat die Auslandsforschung geringere Bedeutung als in der Bundesrepublik Deutschland, und daraus ergibt sich in weiterer Konsequenz, daß ein Großteil des Forschungspotentials dem eigenen Staat bzw. den Ländern und Kantonen erhalten bleibt;

4. woraus sich in weiterer Folge ein wesentlich stärkeres und auch früheres Engagement von etablierten Wissenschaftlern und Nachwuchskräften in der angewandten Forschung im eigenen Land ergeben hat.

Die geographische Forschung wird daher stark geprägt durch die Mitarbeit an geographischen Forschungsprogrammen, damit einer problemorientierten Zusammenarbeit mit anderen Wissenschaftlern, einer Abkehr vom historisch erklärenden bzw. analytischen Denken und einer Hinwendung zu raumordnungspolitischen und handlungsorientierten Perspektiven. Damit hat sich die Arbeit in den sektoralen Subdiszi- plinen, wie der Wirtschafts-, Bevölkerungsgeographie usf., vielfach zu solcher in Problemfeldern verändert. 5. Aufgrund der in beiden Staaten im Vordergrund stehenden Hochgebirgsprobleme wurde schon früh ein beachtlicher Teil der Forschung von den Fragen des physischen Raums absorbiert. Es sei darauf hingewiesen, daß, von H.KINZL in Innsbruck initiiert und in Zusammenarbeit mit dem Deutschen und Österreichischen Alpenverein, regelmäßige Gletschermessungen schon ab der $\mathrm{Z}$ wischenkriegszeit erfolgten und damit die Gletscherforschung stets auch Geographen angezogen hat. Die Klimaforschung besitzt ferner in beiden Staaten echte Schwerpunkte.

6. Beide Staaten sind Bergbauernstaaten. Nicht nur die Abgrenzung des Berggebietes, sondern alle Fragen von Siedlung und Wirtschaft im Hochgebirge haben daher in der Forschung eine weit zurückreichende Vergangenheit.

Die Geographen partizipieren ferner an der räumlichen Disparitätenforschung ebenso wie an der Stadtforschung, wobei allerdings letztere weniger in der Schweiz als in Österreich in der Wiener Schule der Stadtgeographie ein bereits auf Hugo HASSINGER zurückgehendes Zentrum besitzt.

\subsection{Angewandte Forschung an der Universität}

Während die institutionelle Einbindung der angewandten Forschung bei einer marxistischen Staatsdoktrin zentralistisch gelöst wird, stellt sich die Frage, welche Konsequenzen sich im Westen für die Wissenschaft als Organisationsform und als gesellschaftliches Phänomen ergeben, sobald sie sich mit Problemen der angewandten Forschung auseinandersetzt. Diese Frage betrifft ebenso wie die eingangs angeschnittene nicht nur die Geographie, sondern auch andere Disziplinen.

Anhand einer Matrix von Problemstellung und Problemlösung (vgl. Abb.2) sei zunächst davon ausgegangen, welche Forschungsaufgaben an Universitätsinstituten interessant wären. Es handelt sich im wesentlichen um drei Typen:

1. Besonders reizvoll sind Forschungsaufgaben, sobald das Problem selbst erst unscharf definiert ist und die Lösung gesucht werden muß.

2. Im zweiten Fall ist nur die Problemstellung anfangs unklar, sie muß ausformuliert werden, und ebenso muß eine Festlegung von gesellschaftlichen Prioritäten erfolgen, während andererseits die Problemlösungen für diese Alternativen bekannt sind.

3. Es kann die Fragestellung bereits klar definiert sein, während eine Strategie zur Problemlösung gefunden werden muß.

Den theoretischen Überlegungen hinsichtlich «idealer Forschungsaufgaben" stehen zahlreiche organisatorische Schwierigkeiten gegenüber, welche die Chance einer stärkeren Verankerung einer angewandten Forschung an den Universitätsinstituten sehr reduzieren. 


\section{AUFTRAGSFORSCHUNG AN UNIVERSITÄTEN}

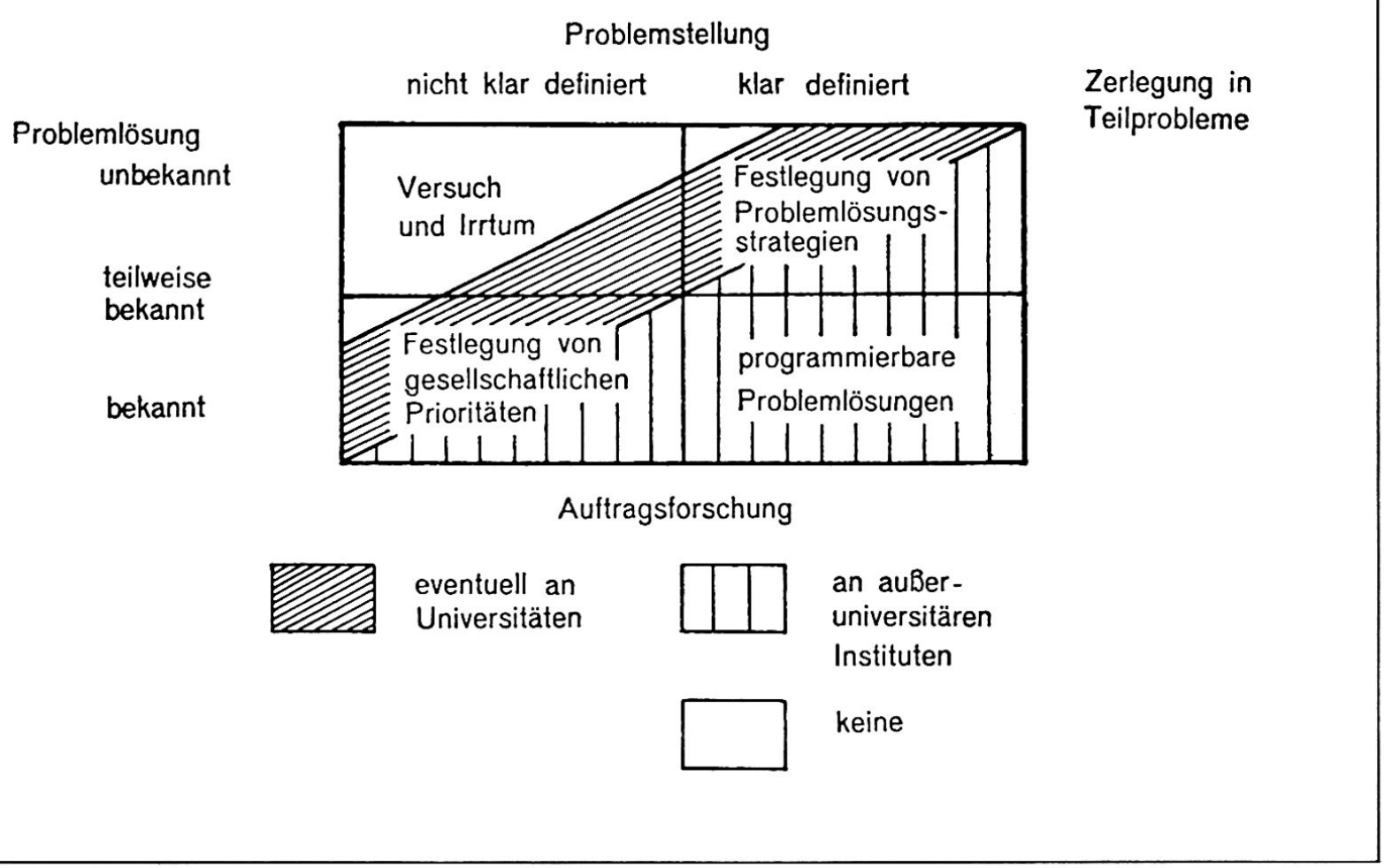

Abb. 2 Die Stellung der Auftragsforschung an Geographischen Universitätsinstituten

Aufgrund von internen Querelen ziemlich unbemerkt, haben sich nämlich in der Nachkriegszeit außerhalb der Universitäten auch in den Sozialwissenschaften große halboffizielle Institutionen entwickelt, und ebenso haben neue Behörden eigene Forschungseinrichtungen aufgebaut, um den wachsenden Bedarf an Begleitforschung zum raschen gesellschaftlichen Wandel abzudecken. Aufgrund dieser Entwicklung werden gegenwärtig Aufträge zumeist nur dann an Universitätsinstitute herangetragen, wenn sie als Reste von Projekten aufgrund ihres geringeren Umfangs bzw. schlecht kalkulierbarer Kosten für arbeitsökonomisch ausgerichtete halboffizielle oder privatwirtschaftliche Forschungsinstitute uninteressant sind.

Ferner darf nicht übersehen werden, daß der durch die Technisierung der Forschung ständig wachsende Kapitalbedarf eine ebenso wachsende Abhängigkeit von den Geldgebern mit sich bringt. Das daraus resultierende Problem der Alibiforschung ist bekannt. Schließlich muß angemerkt werden, daß Wissenschaftlern im allgemeinen die notwendigen Managementerfahrungen im Hinblick auf Arbeitsorganisation und Kostenstruktur fehlen und die Auftragsforschung an den Universitäten keine akademischen Karrieren ermöglicht, so daß darin Beschäftigte diese Tätigkeit nur als Übergangslösung auffassen können.
Überhaupt bedeutet angewandte Forschung über kurz oder lang die Aufgabe der einzelwissenschaftlich organisierten Forschung, und zwar aufgrund des sprunghaft wachsenden Bedarfs an Hilfskräften und damit einer völlig geänderten Situation gegenüber der, wie sie MAX WEBER in seinem Essay "Vom inneren Beruf zur Wissenschaft» beschrieben hat. Nun mag es freilich einzelnen Wissenschaftlern mit organisatorischer Begabung gelingen, eine Art Doppelexistenz zu führen und über Forschungsmittel aus verschiedenen Fonds einen Mitarbeiterstab für bestimmte Projekte aufzubauen. Solange derartige "wissenschaftliche Produktionsstrukturen" jedoch nicht institutionell verankert werden können, sind sie meist nur von kurzer Lebensdauer. Für ein kontinuierliches Management der angewandten Forschung an den Universitäten fehlen im allgemeinen die personellen und finanziellen Voraussetzungen. Vor allem große, langjährige Forschungsvorhaben werden in nicht zu ferner Zukunft nur mehr an großen außeruniversitären Instituten und Forschungseinrichtungen bewältigt werden können.

Hierzu trägt ferner bei, daß zwischen Wissenschaftlern und Vertretern von Körperschaften und Behörden nur unzureichende Kommunikationsmuster bestehen, so daß Aufträge einerseits nach den Prinzipien 
einer informellen Gesellschaft und andererseits nach der reinen Zufallsstreuung vergeben werden. Auch hierbei sind die von der Auftragsforschung lebenden Institutionen, die einen ständigen Kontakt mit den Auftraggebern unterhalten müssen, den Universitäten überlegen.

Als letztes noch einige Aussagen zur gerne zitierten Einheit von Forschung und Lehre. Sie ist in der organisatorischen Praxis nur selten zu realisieren, vor allem deshalb, weil sie voraussetzt, daß erstens ein Lernelement in die Forschung eingebaut wird und zweitens Forschungs- und Lehrniveau auf einer methodischen Ebene liegen. Dies kann ausschließlich bei der Sammlung von standardisierten Informationen der Fall sein, nicht jedoch bei der wissenschaftlichen Weiterverarbeitung der Daten, von den seltenen Fällen abgesehen, wo sich Studenten als künftige Wissenschaftler qualifizieren.

\subsection{Das Ende des institutionellen Wachstums}

Die Geographie konnte als Universitätsdisziplin in den zwei Jahrzehnten der Nachkriegszeit bis herauf zum Wirtschaftseinbruch 1973 in den meisten europäischen Staaten, darunter in der Bundesrepublik Deutschland, in Großbritannien, in der Schweiz und in Österreich, in Frankreich, Spanien und Italien, am Wachstum der Universitäten, gemessen an der Zahl des Lehrpersonals und der Studenten, partizipieren (vgl. Abb. 3). Seither ist das Wachstum weitgehend zum Stillstand gelangt, und es stellt sich die Frage nach den Konsequenzen des Wachstums und dem Strukturwandel unter dem Vorzeichen der Stagnation.

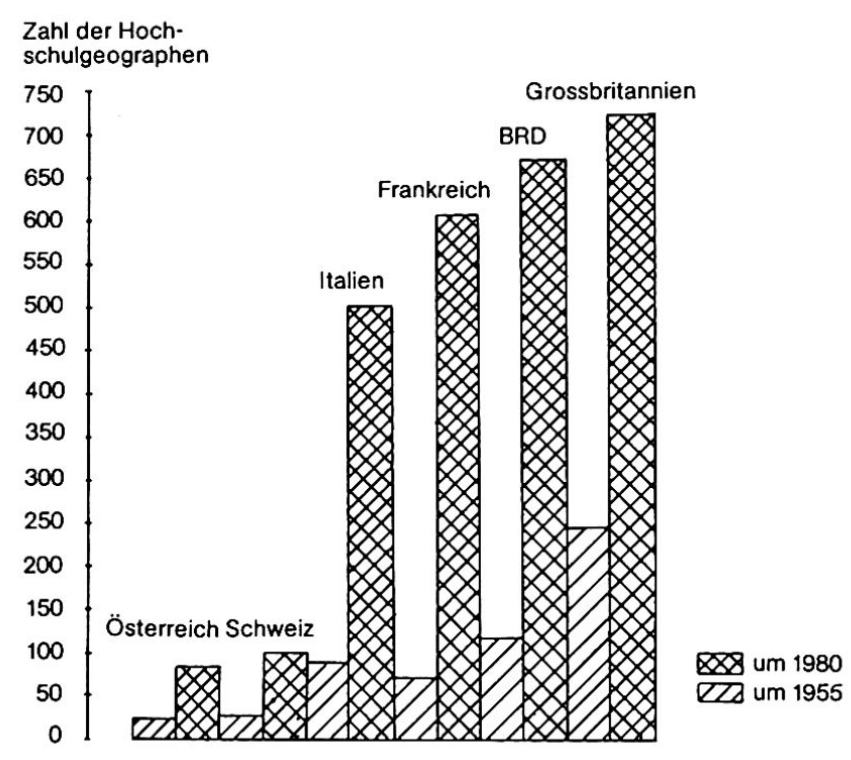

Entwurf: E. Lichtenberger

Abb. 3 Die Zunahme der Zahl der Hochschulgeographen von 1955 bis 1980 in Österreich, der Schweiz, Italien, Frankreich, der Bundesrepublik Deutschland und Großbritannien
1. Durch das Wachstum wurde das fachinterne Kommunikations- und Informationsmuster der Universitätsgeographie grundsätzlich verändert, und $z$ war von zwei Seiten aus: einerseits vom passiven Standpunkt des Informiertseins des einzelnen Wissenschaftlers, und andererseits von der Möglichkeit her, welche der einzelne besitzt, die eigenen wissenschaftlichen Resultate mit anderen zu diskutieren und bekanntzumachen. Die Verfasserin hat in einem früheren Aufsatz den Vorzug der zentralisierten Organisationsstruktur der britischen Geographie herausgestellt und auf das vorbildliche dreistufige System von Veröffentlichungen mit den Zielsetzungen der bibliographischen Information, der zusammenfassenden Übersicht und der Schaffung eines Diskussionsforums hingewiesen, das im Gegensatz steht zum Institutspartikularismus der Publikationen in der Bundesrepublik Deutschland. Es wäre danach anzunehmen, daß eine bessere Organisationsstruktur der Information $\mathrm{zu}$ einem besseren Informiertsein des einzelnen Mitglieds einer bestimmten Disziplin beiträgt. Diese Annahme ist bisher in einem interkulturellen Kontext für die Geographie jedoch nicht durch eine empirische Analyse verifiziert worden. Auf der anderen Seite können wir bei R.JOHNSTON in einem Überblick über die britische Geographie in der Nachkriegszeit nachlesen, daß auch in diesem Staat im Zuge des Wachstums eine geradezu "anarchische» Situation der aktiven Kommunikation eingetreten ist, d.h. die Forschungsstruktur der Geographie zerfallen ist in ein unzusammenhängendes Nebeneinander von Kleingruppen, die ziemlich unabhängig voneinander die "soziale Kontrolle» über das Qualitätsniveau ihrer Mitglieder ausüben. Ein Blick über den Zaun der eigenen Disziplin hinweg belehrt allerdings rasch, daß eine derartige Kleingruppenstruktur auch die benachbarten Disziplinen, wie die Soziologie, Psychologie usf., kennzeichnet, wo ebenfalls bei jeder nationalen und internationalen Tagung stets neue Kleingruppen auftreten.

Besonders eindrucksvoll ist die Abfolge sozialer Kontrollinstanzen in der Universitätsgeographie der Bundesrepublik Deutschland, wo auf die Generation der "Päpste» in den 60er Jahren die in Teildisziplinen etablierten Fachvertreter gefolgt sind, deren Klientelen nun zunehmend von einer Kleingruppenstruktur der Forschung abgelöst werden.

2. In der Phase des Wachstums wurde schließlich eine Hypothek der Altersstruktur des akademischen Lehrkörpers aufgenommen, wie sie aus Abb. 4 anhand der Gegenüberstellung des Lehrkörpers der geographischen Institute in der Bundesrepublik Deutschland um die Mitte der 50er Jahre und um 1980 zu entnehmen ist. Nun liegt es keineswegs in meiner Absicht, die Konsequenzen der Beschränkung der Stellenzahl und der de facto eingetretenen «Schließung der Zunft» zu dramatisieren. Nichtsdestoweniger sollte man die daraus resultierenden Persistenzphänomene in ihrer Langzeitwirkung nicht unterschätzen, wie die 
Festschreibung von wissenschaftlichen Arbeitsrichtungen auf der einen Seite und das Abwandern der Talente an außeruniversitäre Forschungsinstitute bzw. in den privaten und öffentlichen Sektor auf der anderen Seite. Auch andere Disziplinen sind übrigens von beiden Phänomenen, dem Zusammenbruch des Informations- und Kontrollsystems und der Auflösung in eine Kleingruppenstruktur der Forschung sowie der Schließung der Zünfte, ebenso stark betroffen.

3. Mindestens so wichtig ist jedoch das dritte Problem, nämlich die unzureichende Schnittstelle zwischen der Bildungspolitik und der Arbeitsmarktpolitik. Das Wachstum der Universitätsdisziplinen in der 60er Jahren stand noch im Vorzeichen der Bildungsexplosion. Die Reduzierung der Nachfrage nach Lehrern hat die Geographie nun in dieselbe Situation gebracht, in der sich andere Disziplinen, wie die Soziologie, die
Psychologie usf., schon seit langem befinden, nämlich daß sie ihre Studenten nicht auf einen professionalisierten Arbeitsmarkt verweisen kann.

Nun ist die Reduzierung der Nachfrage nach Lehrern keineswegs eine Besonderheit des deutschen Sprachraums. Weit früher mußte und konnte sich die britische Geographie daran anpassen. Details hierzu können einem früheren Aufsatz der Verfasserin (1980) entnommen werden. Der Lehrberuf ist in der Ausbildung in Großbritannien praktisch bedeutungslos geworden, und die Wirtschaft nimmt über die Hälfte der M.A.-Absolventen, d. h. der Diplomanden, auf. In der Bundesrepublik Deutschland ist derzeit eine Umfunktionierung von Ausbildungsplätzen von Lehrern in solche von Diplomgeographen in vollem Gange. Es soll hier nur am Rande auf die Schwierigkeiten hingewiesen werden, daß freigesetzte Lehrka-

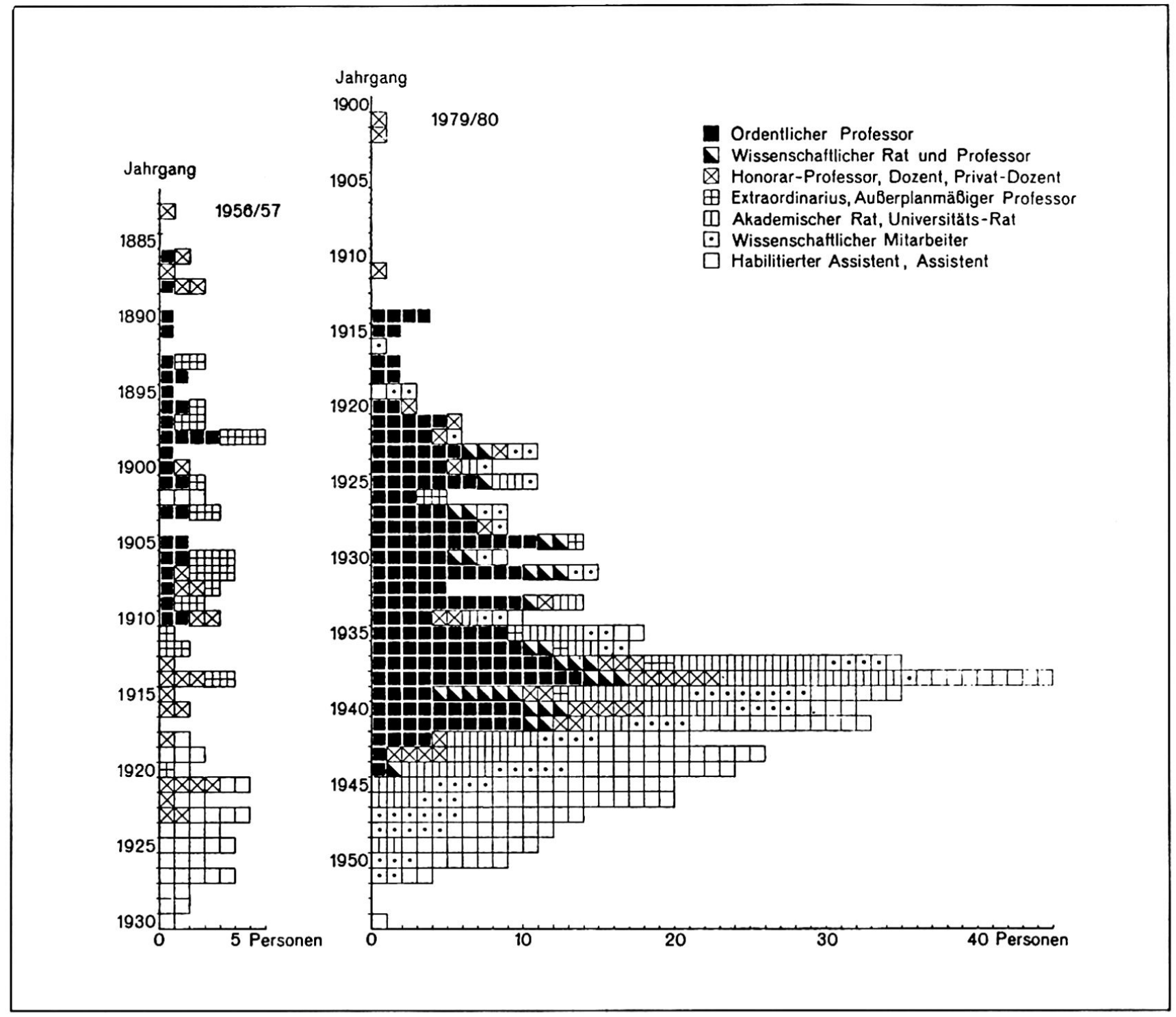

Abb. 4 Die Altersstruktur des Lehrkörpers der Geographischen Institute an Universitäten der Bundesrepublik Deutschland $1956 / 57$ und $1979 / 80$ 


\section{Studienjahr}

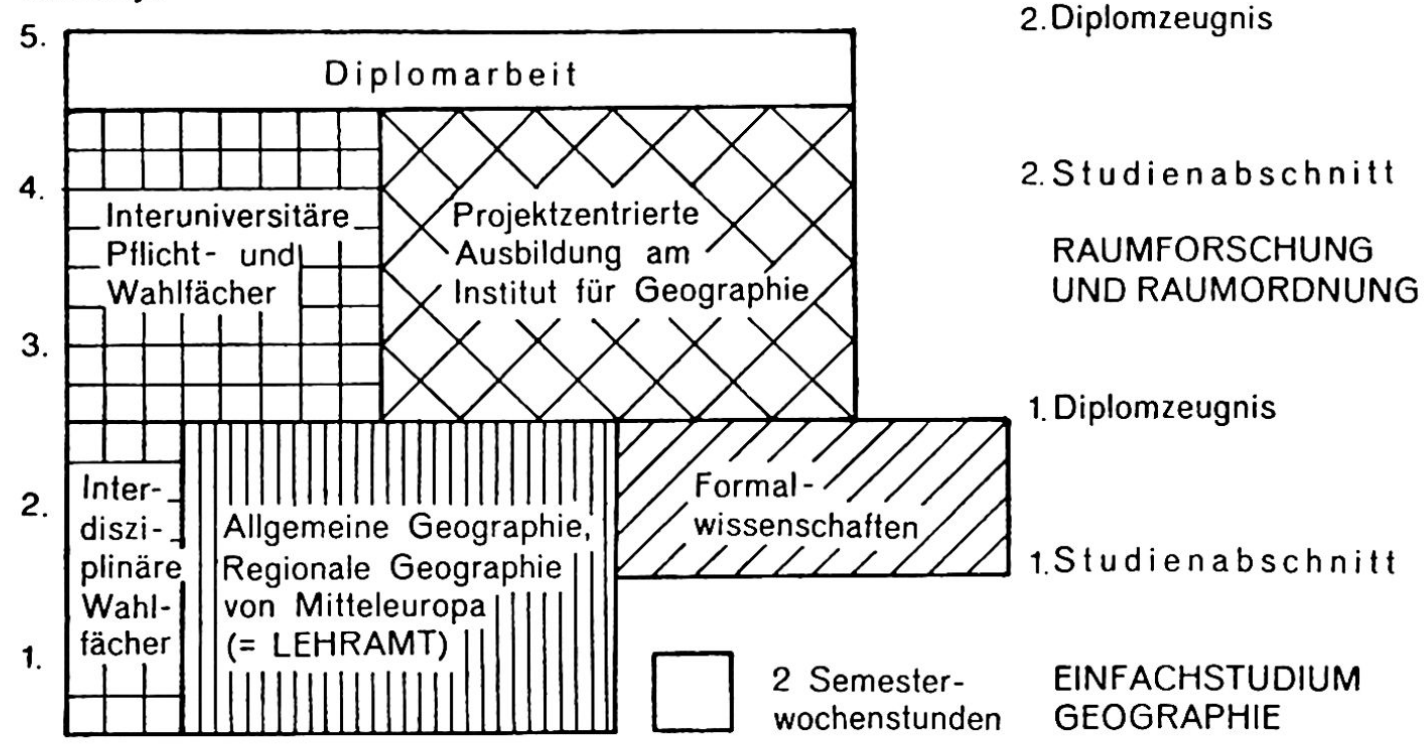

2.Diplomzeugnis

2. Studienabschnitt

RAUMFORSCHUNG UND RAUMORDNUNG

1. Diplomzeugnis

GEOGRAPHIE

Abb. 5 Organisationsschema des Studienzweigs Raumforschung und Raumordnung am Institut für Geographie der Universität Wien

pazitäten, wie sie durch den Rückgang der Lehrerausbildung entstanden sind, bei allem Respekt vor der Reagibilität des Produktionsfaktors Arbeit, keineswegs beliebig umgewidmet werden können. Ebenso erscheint es schwierig, Ausbildungsinhalte im Hinblick auf die Planungspraxis oder Wirtschaft $\mathrm{zu}$ formulieren, wenn die Ausbildenden selbst weder in dem einen noch in dem anderen Bereich tätig waren.

In diesem Zusammenhang mag es von Interesse erscheinen, die Wiener Lösung vorzuführen. Das Geographische Institut der Universität Wien ist in der glücklichen Lage, schon vor der Absatzkrise für Lehrer zwei Studienzweige, nämlich Raumforschung und Raumordnung sowie Kartographie, eingerichtet zu haben (1974), ohne daß von seiten der Nachfrage ein Druck ausgeübt worden wäre. Beide Studienzweige sind institutionell in der Geographie verankert, sachlich jedoch interdisziplinär aufgebaut. Aus dem Organisationsschema für den Studienzweig Raumforschung und Raumordnung (vgl. Abb. 5) ist zu entnehmen, daß im ersten Studienabschnitt etwa $40 \%$ des Lehrangebots mit dem für Lehramtskandidaten identisch, weitere $40 \%$ durch formalwissenschaftliche Übungen und Vorlesungen besetzt, ca. 20\% als interdisziplinäre Wahlfächer, wie Volkswirtschaftslehre und Betriebswirtschaftslehre, deklariert sind.

Der zweite Studienabschnitt ist projektzentriert. In jedem Semester findet ein Forschungsprojektseminar statt, zu dem in Ergänzung Veranstaltungen von auswärtigen Lektoren, darunter von Planungsexperten, angeboten sowie Lehrveranstaltungen und Seminare an anderen Universitäten besucht werden. Aufgrund der bisher nur kleinen Zahl von Absolventen bestehen keine Schwierigkeiten, interessante berufliche Positionen bei Forschungsinstituten, Behörden und in der Privatwirtschaft zu finden.

\section{Zur Struktur und Problematik der geographischen Forschung}

\subsection{Die Forschungstypologie im dreidimensionalen Informationsraum der Geographie}

Das Informations- und Forschungsterrain der Geographie wird durch drei Dimensionen definiert, d. h. nach

- sachlichen Inhalten (S)

- Theorien und Methoden (M) sowie nach der

- räumlichen Bezugsebene (R) (vgl. Abb. 6).

Die Geographie und ihre Subdisziplinen befinden sich daher gegenüber den systematischen Disziplinen im Nachteil, deren Mitglieder im allgemeinen mit einem zweidimensionalen Informations- und Forschungsraum ihr Auslangen finden.

Nun ist der dreidimensionale Informations- und Forschungsraum in der Geographie insgesamt äußerst ungleichmäßig besetzt. Es klaffen Leerräume, die als 
Unterbrecher fungieren, andererseits kommt es zu Ballungen und damit $\mathrm{zu}$ einer Intensivierung der Kontakte. Diese unscharf formulierte Annahme beruht auf der intellektuell stimulierenden Analyse von Zitierkartellen von G.HARD, durch welche allerdings nur ein schmales Literatursegment aufgeschlossen wurde.

Aufgrund von Biographien wird im folgenden versucht, in idealtypischer Weise diejenigen Verschiebungen im dreidimensionalen Forschungsterrain herauszustellen, welche am häufigsten auftreten, und danach Forschertypen zu kreieren:

1. An erster Stelle sei der territoriale Forscher genannt. $\mathrm{Er}$ ist dadurch gekennzeichnet, daß er die räumliche Bezugsebene in seinem wissenschaftlichen Leben weitgehend beibehält und in der spezifischen Region (Stadt) verschiedene Sachinhalte mit gleicher oder wechselnder Methodik untersucht. Dabei kommt es zwangsläufig zu starken Kontakten mit Vertretern anderer Fachdisziplinen und über die angewandte
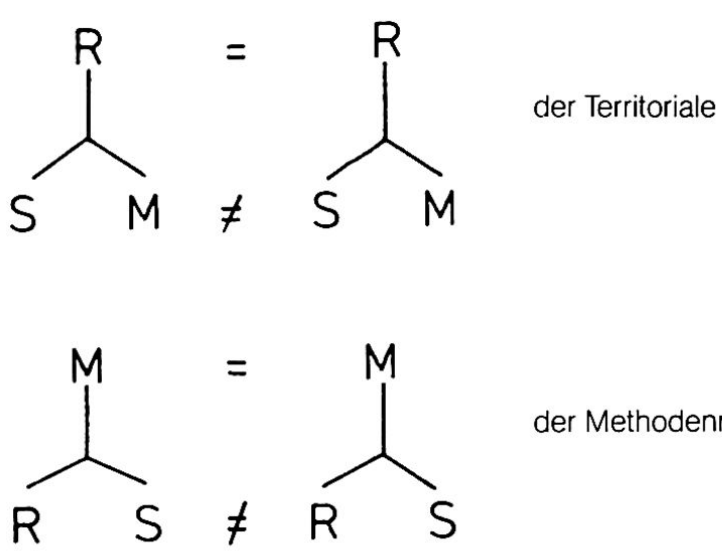

der Methodenmonist<smiles>[R]C(S)SC</smiles>

der komparative Sachkenner

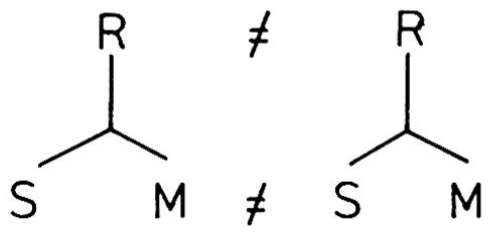

der Rösselspringer

$R$ regionaler Bezug

$S$ sachliche Fragestellung

$M$ Methoden (und Theorien)

Entwurf: E. Lichtenberger

Abb. 6 Die Typologie der Forscher im dreidimensionalen Informationsraum der Geographie
Forschung zu solchen mit Behördenvertretern. Wenn diese externe Orientierung die Oberhand gewinnt, so lockern sich die Kontakte zu den Fachkollegen.

2. Als Gegenpol zum Territorialen kann der Methodenmonist angesehen werden, der Sachinhalte und räumliche Bezugseinheiten wechselt, seine spezialisierte Methodik (Theorie) jedoch beibehält und sich in dieser vielfach an der Forschungsfront bewegt. Sehr viele Vertreter des angelsächsischen Sprachraums, ebenso aber auch Mitglieder des Arbeitskreises für Theorie und quantitative Methodik in der Geographie haben einen begreiflichen Hang zum Methoden(Theorie-)monismus. Die zahlreichen Kontakte sind zumeist interdisziplinär, die Methodik dient als Verbundschiene, welche den Standortwechsel in sachlicher und regionaler Hinsicht sehr erleichtert. Häufig - und hierfür stehen vor allem Vertreter aus der angelsächsischen Welt - lockern sich die Bezüge zum Realobjektraum, die Perfektion der Methodik wird zu einer l'art pour l'art, die Modelle erhalten platonischen Charakter, des öfteren wird vergessen, daß die Geographie eine empirische Disziplin darstellt.

3. Der dritte Typ wird als komparativer Sachspezialist bezeichnet. Er widmet sich einer bestimmten kleineren oder größeren Fragestellung, die er am besten mit einer bestimmten Methode lösen kann, und wechselt mit beiden das Gebiet. Dieser sehr häufige Typ trägt zur Aufsplitterung der Geographie in immer weitere Subdisziplinen und Themenkreise bei. Öfters trägt er seine Spezialisierung, ähnlich dem Methodenmonisten, wie die Schnecke das Gehäuse recht unbekümmert über Kontinente hinweg mit sich.

4. $\mathrm{Zu}$ einer im Aussterben begriffenen Spezies zählt der Rösselspringer. Er wechselt, sei es aus persönlichem Plaisir, sei es durch die Karrierenormen gezwungen, durch Aufträge oder was immer veranlaßt, nicht nur Sachfragen und Methodik, sondern gleichzeitig auch das Gebiet und gelangt damit in immer neue Informations- und Diskussionskreise. Gerade daraus mag erneut ein Impetus für einen weiteren Rösselsprung gewonnen werden. Aus der bereits in die Wissenschaftsgeschichte eingegangenen Generation kann Hans вовек genannt werden, der sich außer mit der Sozialgeographie und dem Rentenkapitalismus (im Vorderen Orient) mit der Quartärforschung in den Alpen und im Iran und ebenso mit klimaökologischen Fragen beschäftigt hat.

Selbstverständlich lassen sich diese vier Grundtypen beliebig weiter aufspalten. In der bundesdeutschen Geographie läßt sich z. B. ein fünfter Typ anführen, der mit zwei regionalen Standbeinen ausgestattete Forscher, der im Umfeld seiner Universitätsstadt und im Ausland arbeitet, wobei allerdings das Fragen- und Methodenset im allgemeinen erst im Inland erprobt wird, bevor seine Anwendung im Ausland erfolgt. 


\subsection{Geographischer Maßstab und Theoriehorizonte}

Anhand eines einfachen Schemas (vgl. Abb. 7) sei das Problem des geographischen Maßstabs und sein Verhältnis zu den aktuellen Theoriehorizonten von Nachbardisziplinen der Geographie skizziert. Es wird hierbei von der Annahme ausgegangen, da $B$ sich alle systematischen Disziplinen im Laufe ihrer Entwicklung vom Makromaßstab zum Mikromaßstab hin bewegen. Ohne diese sachspezifischen Maßstäbe nun näher in Skalenwerten definieren zu wollen, wurden von rechts unten nach links oben die wichtigsten systematischen Wissenschaften eingetragen, wobei die Pfeile die räumliche Ebene definieren, in der die einzelnen Disziplinen arbeiten. Der geographische Maßstab wird in diesem Schema mit dem Landschaftsbegriff identifiziert (in der Humangeographie Parzelle und Haus als untere Einheit). Er bildet einen einheitlichen vertikalen Streifen, der die horizontal abgebildeten räumlichen Ebenen der systematischen Fächer schneidet und folgendes Problem offenlegt: In der physischen Geographie bewegen sich alle
Nachbardisziplinen, wie die Vegetationsgeographie, Geologie, Petrographie, Mineralogie, Bodenkunde, bereits gegen den Mikromaßstab hin. Ihre räumliche Bezugsebene bei der empirischen Analyse liegt damit vielfach tiefer als die der angrenzenden geographischen Fächer. Einige haben aber einmal in demjenigen Maßstab gearbeitet, in dem sich letztere Fächer gegenwärtig befinden. Es bedeutet daher für die geographischen Fächer keine sonderliche Schwierigkeit, theoretisch und methodisch an die Nachbardisziplinen anzuschließen, wobei sie überall dort, wo sich ihre systematischen Kontrahenten bereits auf den Mikromaßstab zu bewegen, das von diesen geräumte Forschungsterrain besetzen (z. B. Witterungsklimatologie, Pflanzengeographie). Diese Feststellung schließt an die Aussage von G.HARD an, daß grundsätzlich im Konnex von sachlichem und räumlichem Bezug zwischen Theorien, Methoden und Fragestellungen, die von Nachbarwissenschaften verwendet werden, und denjenigen der entsprechenden Subdisziplinen der Geographie keine Unterschiede bestehen. Dieser Aussage ist zuzustimmen, mit einer Ausnahme: Im

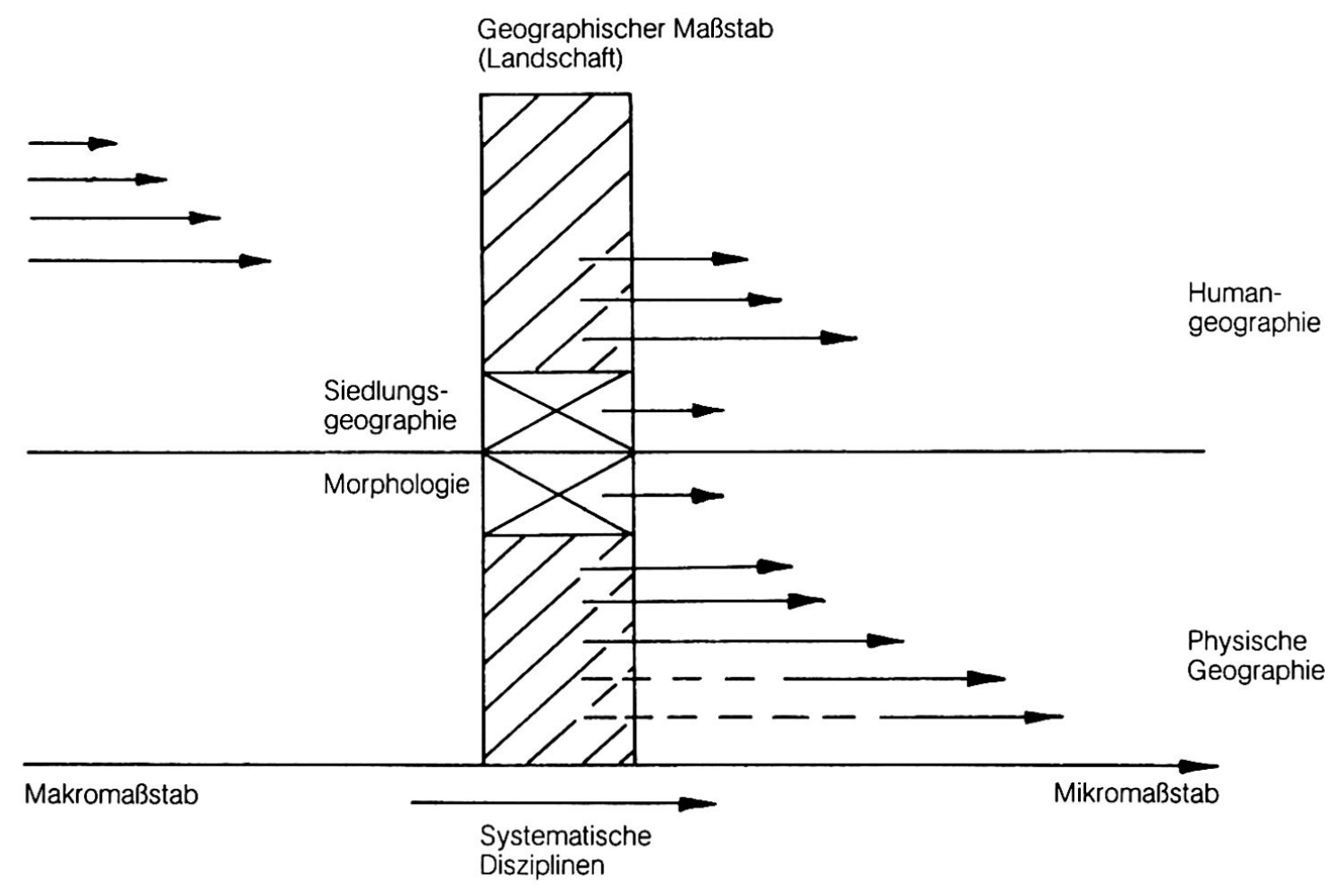

aktueller Theoriehorizont

Entwurf: E. Lichtenberger

Abb. 7 Geographischer Maßstab und aktuelle Theoriehorizonte von Nachbardisziplinen der Geographie 
Rahmen der physischen Geographie hat sich die Morphologie schon früh von der Geologie separiert, ein eigenes Klassifikationssystem, eigene Theorien und eine eigene Aufgabenstellung entwickelt.

Wenden wir uns den Humanwissenschaften zu, so besteht hier hinsichtlich des Theoriehorizonts eine deutliche Zweiteilung. Zwei Disziplinen, nämlich die Volkskunde und die Völkerkunde, habe sich schon seit längerem unterhalb der von der Humangeographie untersuchten Maßstabsebene des Realobjektraums und Aktionsraums angesiedelt.

Im Zuge des Take-offs der analytischen Geographie ist nun eine zweite Gruppe von Nachbarwissenschaften in engen Kontakt mit der Geographie geraten, nämlich die Soziologie, die Ökonomie und die Politologie. Ihre Theoriehorizonte liegen derzeit noch über dem Landschaftsmaßstab der Geographie. Im Zuge der generellen Wissenschaftsentwicklung folgen jedoch auch sie dem Trend zur Forschungsarbeit in kleineren Räumen. In diesem Zusammenhang hat sich aus der Makroökonomie die Regionalökonomie entwickelt und ist in der Soziologie als Geo-Ast die Sozialökologie entstanden. Es ist wohl nur eine Frage der Zeit und ziemlich sicher zu erwarten, daß die Geo-Äste der genannten Nachbardisziplinen tiefere räumliche Bezugsebenen erreichen werden. Damit wird ein Druck auf die benachbarten geographischen Teildisziplinen ausgeübt, deren gegenwärtiges Problem darin besteht, daß sie - wie die Wirtschaftsgeographie und teilweise auch die Sozialgeographie -, um den vielzitierten und erwünschten "Theorieimport» von den Nachbarwissenschaften überhaupt vornehmen zu können, den geographischen Maßstab verlassen und eine höhere räumliche Bezugsebene als Forschungsebene wählen müssen, und zwar diejenige, in der diese Nachbarwissenschaften operieren.

Mit dieser Abbildung wurde das Dilemma und gleichzeitig das immanente Problem der Forschungsstruktur der Geographie offengelegt, in der die einzelnen Teildisziplinen aufgrund des notwendigen Konnexes mit den Nachbarwissenschaften in verschiedenen Maßstäben arbeiten müssen.

Eine Sonderstellung unter den humangeographischen Subdisziplinen nimmt die Siedlungsgeographie ein, zu der sich keine Nachbarwissenschaft, sondern nur eine Art «Siedlungslehre» als Propädeutik an den Technischen Universitäten entwickelt hat. Ebenso wie die Morphologie hat auch die Siedlungsgeographie, insbesondere im ländlichen, aber auch im städtischen Raum, eigene Klassifikationssysteme und hermeneutische Theoriehorizonte aufzuweisen.

\section{Quo vadis?}

Blenden wir die institutionellen Aussagen über die Geographie als Universitätsdisziplin mit denen über die pluralistische Forschungsstruktur des Faches zusammen, so gelangen wir zu folgenden Thesen:
1. Es sind normative Zielsetzungen, d.h. "Vorgaben von seiten der Gesellschaft), notwendig, um physische und humanwissenschaftliche Subdisziplinen zu einer integrierten Forschung zu führen. Wie immer die konfliktträchtigen Problemfelder dann auch heiBen mögen, welche die Gesellschaft in ihrem Verhältnis zum Naturraum stets neu definieren wird, so ist jedoch eines sicher, nämlich daß nur der Realobjektraum, in dem die Entscheidungen von seiten der politischen Exponenten und von seiten der privaten Wirtschaftsträger fallen, die Plattform darstellt, auf der wissenschaftliche Ergebnisse als Grundlagenforschung oder angewandte Forschung angeboten werden können.

Ohne die Legitimität der sektoralen Forschung in Frage stellen zu wollen, sei daher die These gestattet, daß Geographie als physische und humane Subdisziplinen umgreifender Forschungsbereich nur dort entstehen kann, wo externe Faktoren einen Push in dieser Richtung ausüben, seien es nun internationale Programme, wie das Man and Biosphere-Programm, oder gesellschaftliche Aufträge.

2. Die äußerst diversifizierte sektorale Forschung ist institutionsintern nicht mehr in übergreifender Weise organisierbar, außer dort vielleicht, wo - dies ist jedoch ein seltener Zufall - sehr gute persönliche Kontakte zwischen Fachvertretern bestehen.

3. Der vielzitierte geographische Maßstab ist selbst als heuristisches Prinzip nicht mehr brauchbar, wenn man von den traditionellen Kerndisziplinen des Faches, der Morphologie und der Siedlungsgeographie, absieht.

4. Was hat die Geographie nun anzubieten gegenüber der Konkurrenz der Nachbardisziplinen, wenn sie dem aktuellen Trend zur angewandten Forschung folgt? Der überwiegend pessimistischen Auffassung vieler Kollegen sei hier eine positive Aussage gegenübergestellt: Dort, wo Kartierung und Geländeerhebung als klassische geowissenschaftliche Methoden mit Luftbildinterpretation und Befragungen zu neuen Landinformationssystemen in verschiedenen Maßstäben verbunden werden, dort, wo die Methode der Mehrebenenanalyse eingesetzt wird, Theorien in verschiedenen räumlichen Maßstäben übergreifend über die Sachverhalte zum Tragen kommen, dort hat die Geographie eine echte Chance gegenüber den Sozial- und Wirtschaftswissenschaften, da sie ein sehr viel besseres Arsenal von Begriffen und Methoden bereithält, um Analysen zu den Konflikten und Entscheidungen über die immer knapper werdende Ressource Raum anzubieten.

Kommen wir zum Schluß:

Wir müssen zur Kenntnis nehmen, daß die Geographie, welche in der Zeit des Bildungsbürgertums die Eliten für ihre weltweiten Forschungen interessieren 
und ihre Forschungsinhalte in Lehrinhalte umsetzen konnte, welche zur Allgemeinbildung gehörten, sich nunmehr an die Konsumgesellschaft anpassen muß. Auf die Vernichtung des Distanzfaktors unter dem Einfluß der Massenmedien, die Anhäufung von punktuellem Wissen wurde hingewiesen. Die Bildungsziele für eine Konsumgesellschaft müssen daher auch neu definiert werden. Die Umpolung von der regionalen Geographie zum Set von Fallstudien hin kann als eine Form der didaktischen Anpassung aufgefaßt werden.

Separiert von den Bildungszielen des Faches entwikkelt sich die sektorale Forschung in den einzelnen Subdisziplinen in eine immer weitere Spezialisierung hinein. Der ins "frische Holz", einem Bohrwurm vergleichbar, vordringende Spezialist hat vielfach bereits den Konnex mit den etablierten Schwerpunkten der Forschung verloren.

In einer Zeit zunehmenden Einflusses des Staates auf alle Ebenen des Lebens, einer immer knapper werdenden Ressource des Raumes, größer werdenden Konflikten zwischen den einzelnen Teilen der Gesellschaft bedarf es jedoch einer angewandten Forschung, welche nicht nur die oligarchischen Strukturen von Organisationssystemen der Privatwirtschaft und des Staates und die daraus resultierenden Konflikte untersucht, sondern sich mit allen Fragen beschäftigt, welche durch die immer stärkere Inanspruchnahme des Realobjektraumes, zuerst von seiten der arbeitsteiligen und heute der der Freizeitgesellschaft, entstehen. Die Universität ist sicher auf die Dauer nicht imstande, diesen Forschungsansprüchen zu genügen. Raumordnungsinstitutionen halboffizieller und offizieller Art sind außerhalb entstanden. Universitätsintern stellen sich neue Forderungen an die Lehrinhalte. In welchem Umfang die Geographie als Hochschulinstitution in Lehre und Forschung an der neuen mächtigen Tendenz einer Begleitforschung zum Wandel der Gesellschaft im Raum partizipieren kann und wird, ist offen und sicherlich gerade in kleinen Staaten von den Personen abhängig, die das Fach an den Hohen Schulen vertreten.

\section{Zitierte Literatur}

ARNBERGER, E. (1982): Das Fehlen richtiger räumlicher Lageund Entfernungsvorstellungen bei den Absolventen Allgemeinbildender Höherer Schulen. Mitt. d. Österr. Geographischen Gesellschaft 124: 184-202.

ARNBERGER, E. und KELNHOFER, E. 1983. Das Ordinariat für Geographie und Kartographie und der Studienzweig Kartographie an der Universität Wien. Beitr. a. d. Seminarbetrieb und Arbeitsbereich d. Ordinariats f. Geographie u. Kartographie 14. Institut für Geographie, Wien.

CLAVAL, P., 1984. France. In: Geography since the Second World War. An International Survey, ed. by R.J. Johnston and P. Claval: $15-41$.

CORI, B., 1984. Italy. In: Geography since the Second World War. An International Survey, ed. by R.J. Johnston and P. Claval: 42-63.

DEUTSCHER VERBAND FÜR ANGEWANDTE GEOGRAPHIE e.V. (Hsg.), 1985. Der Geograph und sein Markt. Qualifikationen, Berufsfelder, Arbeitsmarkt. Material zum Beruf des Geographen 7. Bochum.

GEOGRAPHICA HELVETICA 1984/2. Heft zum Themenschwerpunkt "Angewandte Geographie in der Schweiz". Zürich.

HARD, G. und FLEIGE, H., 1977: Zitierzeiten und Zitierräume in der Geographie. Eine Studie zum Zitierverhalten in der methodologischen Literatur. Mitt. d. Österr. Geographischen Gesellschaft 119: 3-33.

JOHNSTON, R.J. and GREGORY, S., 1984. The United Kingdom. In: Geography since the Second World War. An International Survey, ed. by R.J. Johnston and P. Claval: 107-131.

LICHTENBERGER, E., 1978. Klassische und theoretisch-quantitative Geographie im deutschen Sprachraum. Berichte zur Raumforschung und Raumplanung, Wien, 22/1: 9-21.

LICHTENBERGER, E., 1979: The Impact of Political Systems upon Geography: the Case of the Federal Republic of Germany and the German Democratic Republic. Professional Geographer 31/2: 201-211.

LICHTENBERGER, E., 1980. Zur Standortbestimmung der Universitätsgeographie. Reflexionen über die institutionelle Situation in der BRD und in Großbritannien. Mitt. d. Österr. Geographischen Gesellschaft 122/1: 3-48.

LICHTENBERGER, E., 1984. The German-speaking Countries. In: Geography since the Second World War. An International Survey, ed. by R.J. Johnston and P. Claval: 156-184. 